Supporting Information

\title{
Laser Flash Photolysis Studies on Radical Monofluoromethylation by (Diarylamino)naphthalene Photoredox Catalysis: Long Lifetime of the Excited State is not Always a Requisite
}

\author{
Naoki Noto, ${ }^{\ddagger}$ Keigo Takahashi, ${ }^{\ddagger}$ Shion Goryo, ${ }^{\S}$ Akira Takakado, ${ }^{\S}$ Koichi Iwata, ${ }^{\S}$, Takashi Koike ${ }^{*},{ }^{\ddagger}$ \\ Munetaka Akita*ł‡ \\ †Laboratory for Chemistry and Life Science, Institute of Innovative Research \\ ¥School of Materials and Chemical Technology, Tokyo Institute of Technology \\ R1-27, 4259 Nagatsuta-cho, Midori-ku, Yokohama 226-8503, Japan \\ §Department of Chemistry, Faculty of Science, Gakushuin University, 1-5-1 Mejiro, Toshimaku, Tokyo \\ 171-8588, Japan
}

Table of Contents

Reaction Apparatuses

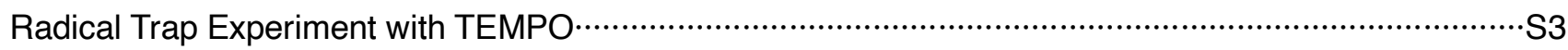

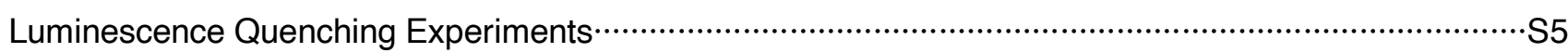

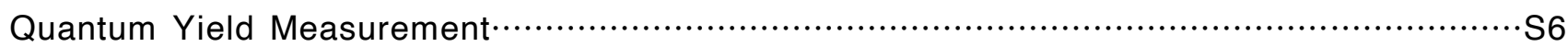

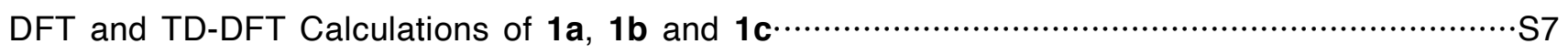

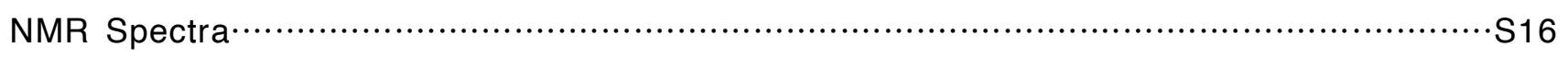




\section{Reaction Apparatuses}

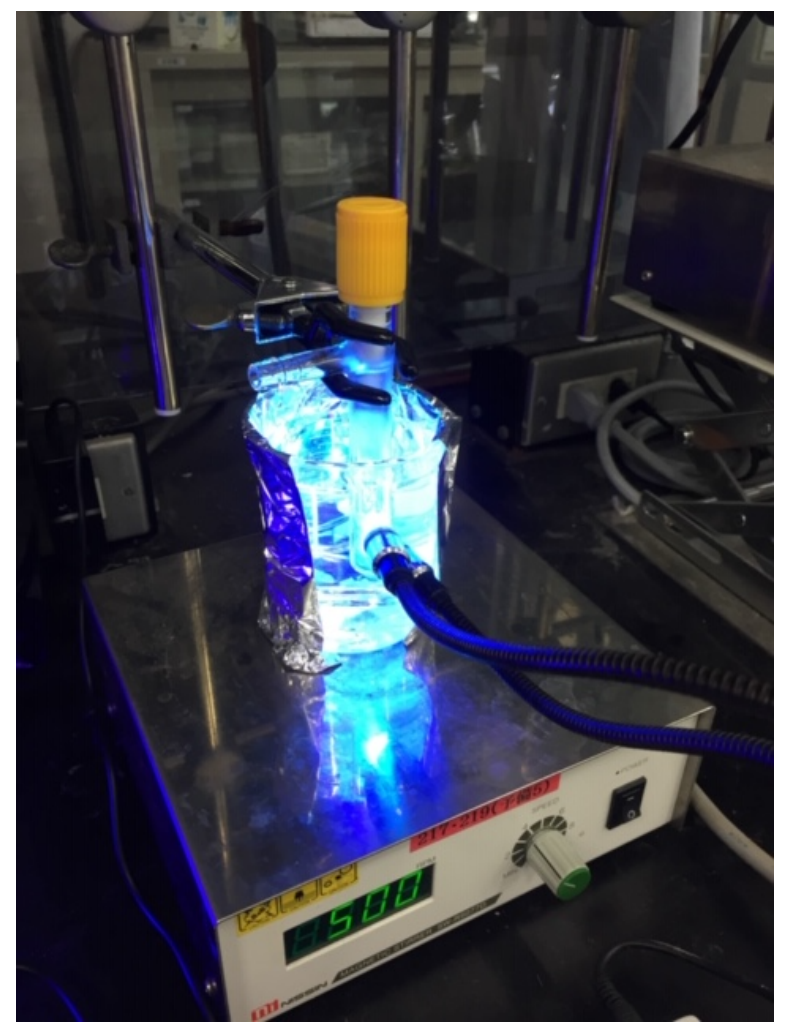




\section{Radical Trap Experiment with TEMPO}
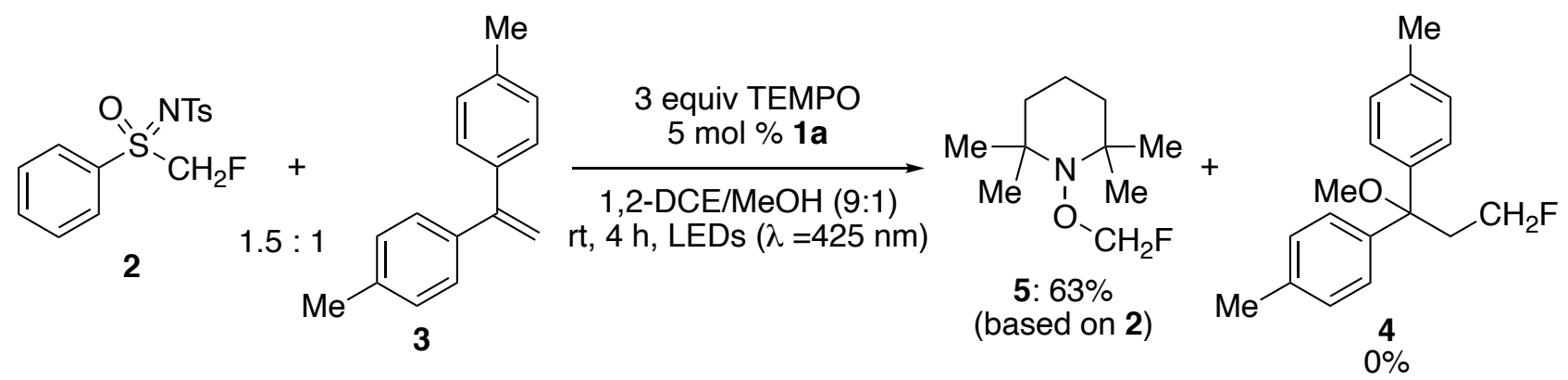

GC-MS
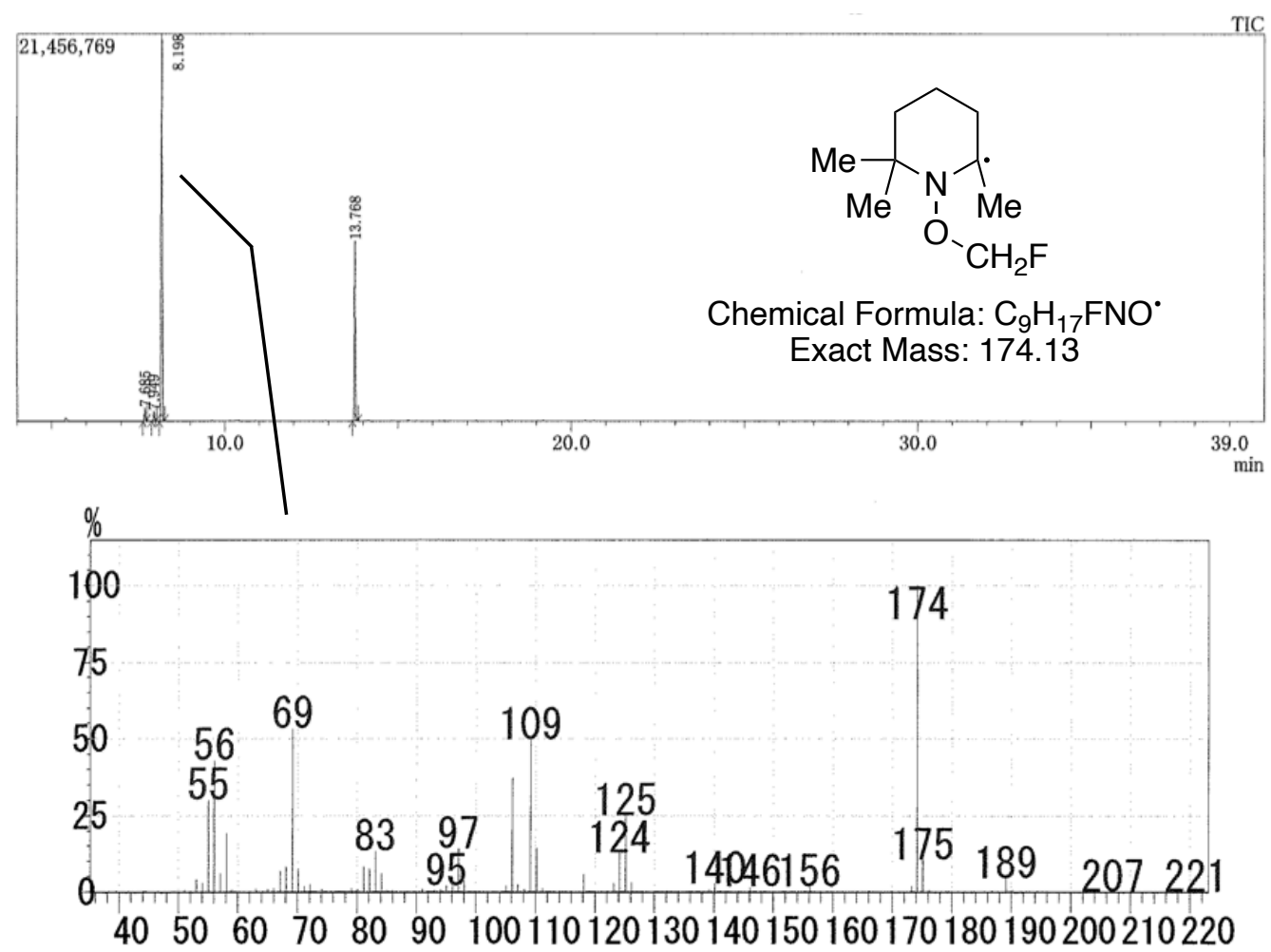

Figure S1. A GC chromatogram and a MS chart. 
${ }^{19} \mathrm{~F} \mathrm{NMR}\left(376 \mathrm{MHz}, \mathrm{CDCl}_{3}, \mathrm{rt}\right)$

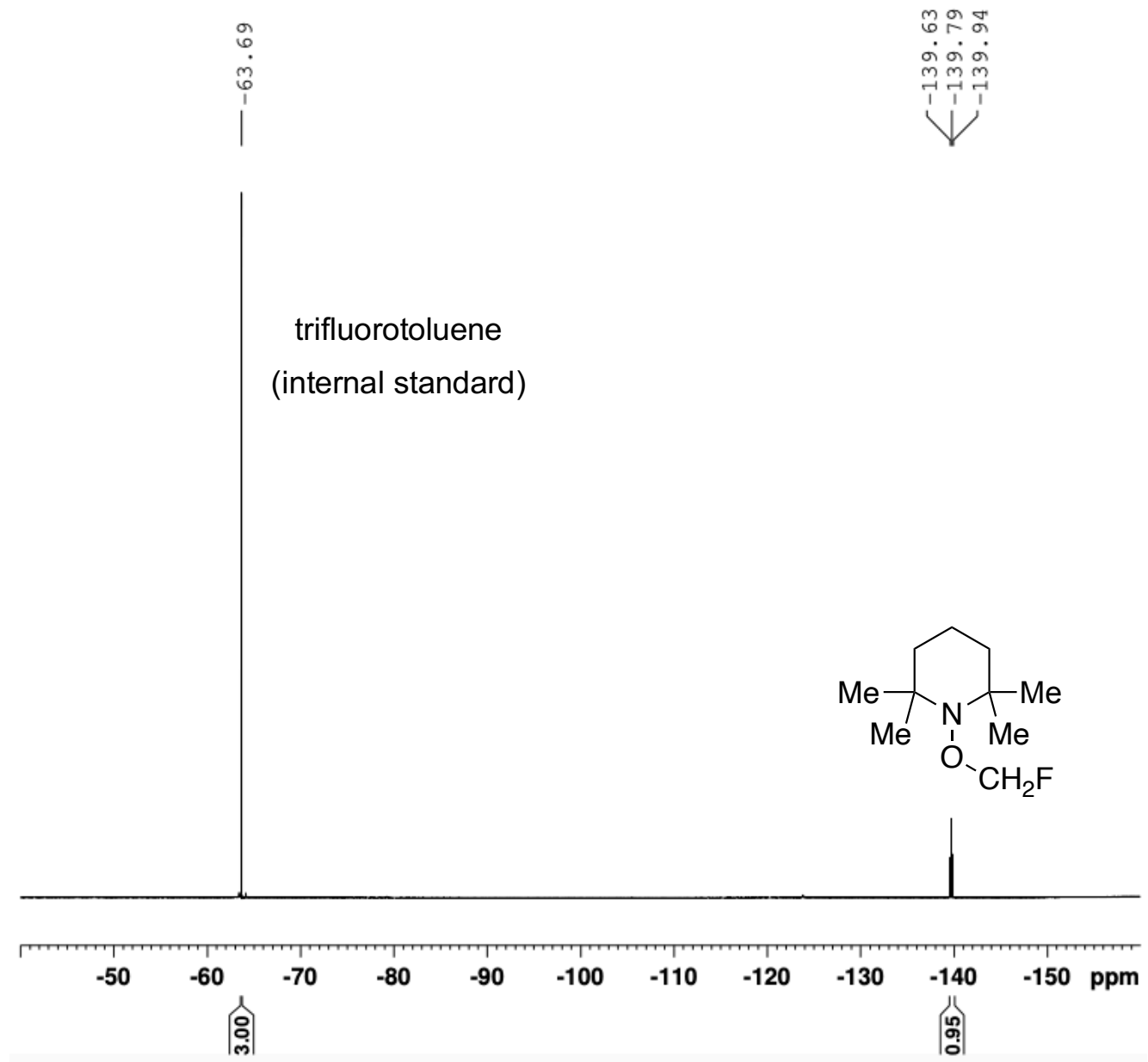

Figure S2. ${ }^{19} \mathrm{~F}$ NMR spectra. 


\section{Luminescence Quenching Experiments}

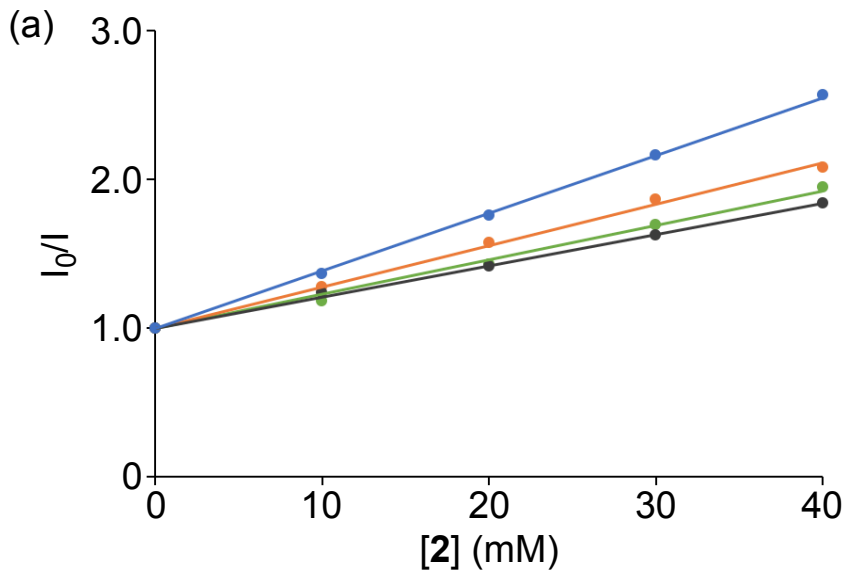

$$
\begin{aligned}
& -\mathbf{1 b} \\
& y=0.0388 x+1 \\
& R^{2}=0.9994 \\
& -\mathbf{N} \\
& y=0.0278 x+1 \\
& R^{2}=0.9970 \\
& -1 a \\
& y=0.0230 x+1 \\
& R^{2}=0.9935 \\
& - \text { fac-[Ir(ppy) } 3] \\
& y=0.0211 x+1 \\
& R^{2}=0.9982
\end{aligned}
$$

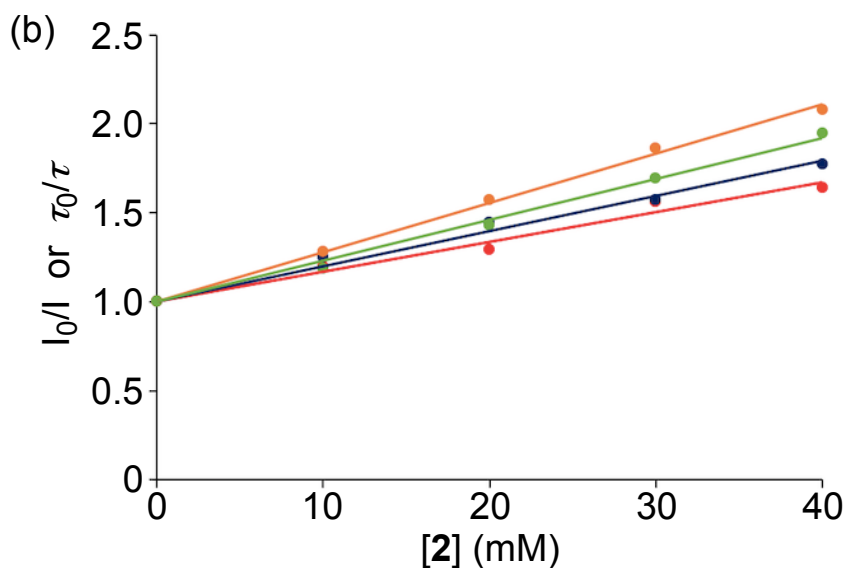

$$
\begin{gathered}
-\mathrm{I}_{0} / \mathrm{l} \text { of } \mathbf{N} \\
\mathrm{y}=0.0278 \mathrm{x}+1 \\
\mathrm{R}^{2}=0.9970 \\
-\mathrm{I}_{0} / \mathrm{l} \text { of } 1 \mathrm{a} \\
\mathrm{y}=0.0230 \mathrm{x}+1 \\
\mathrm{R}^{2}=0.9935 \\
-\tau_{0} / \tau \text { of } 1 \mathrm{a} \\
\mathrm{y}=0.0199 \mathrm{x}+1 \\
\mathrm{R}^{2}=0.9826 \\
-\tau_{0} / \tau \text { of } \mathbf{N} \\
\mathrm{y}=0.0168 \mathrm{x}+1 \\
\mathrm{R}^{2}=0.9753
\end{gathered}
$$

Figure S3. Stern-Volmer plots. (a) $I_{0} / /$ of $\mathbf{1 a}, \mathbf{1 b}, \mathbf{N}$ and fac-[Ir(ppy) $)_{3}$. (b) $I_{0} / l$ and $\tau o / \tau$ of $\mathbf{1 a}$ and $\mathbf{N}$.

(a) $\mathbf{N}$

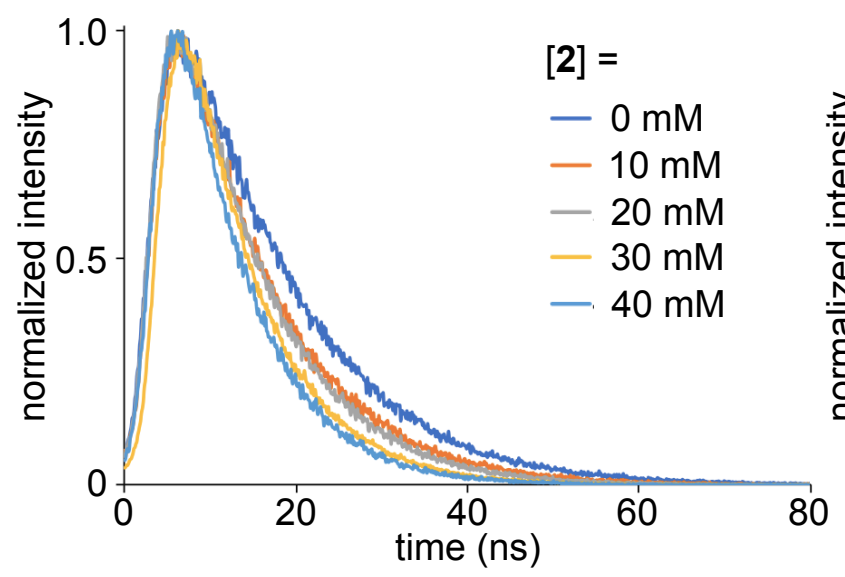

(b) $1 \mathrm{a}$

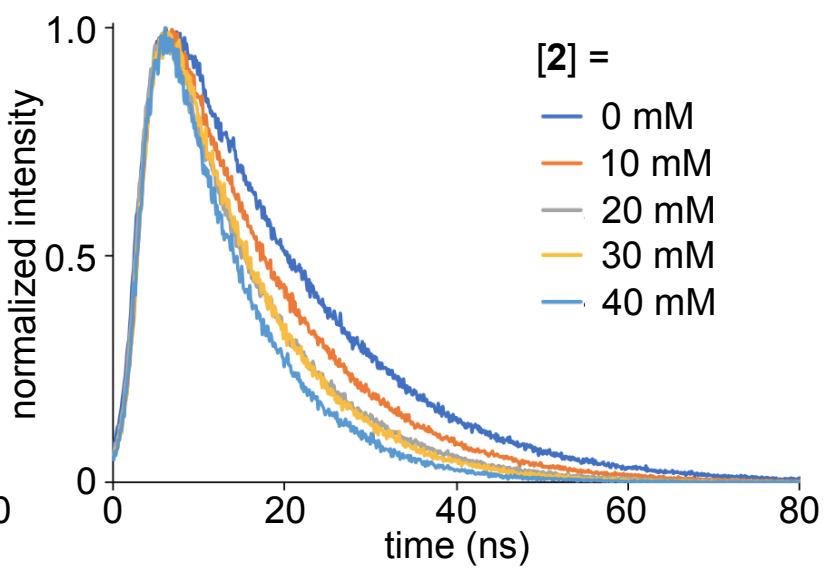

Figure S4. Luminescence decay of (a) N, (b) 1 a. 


\section{Quantum Yield Measurement}

Quantum yield measurement was carried out in the same way to previous our report. ${ }^{[2]}$

(Photon flux)

Photon flux was measured with Ophir PD300-UV and Ophir StarLite.

Irradiation was carried out with HITACHI F-7000 $(\lambda=436 \mathrm{~nm}$, emission slit width $=10.0 \mathrm{~nm})$.

Measured power of light: $115.2 \mu \mathrm{W}$ (for $\lambda=436 \mathrm{~nm}$ )

$$
\begin{aligned}
\text { photon flux }= & \frac{\text { power of light }(\mathrm{W}) \times \text { wavelength of irradiation light }(\mathrm{m})}{\text { Plank constant }(\mathrm{J} \cdot \mathrm{s}) \times \text { speed of light }(\mathrm{m} / \mathrm{s}) \times \text { Avogadro constant }(1 / \mathrm{mol})} \\
& \therefore \frac{115.2 \times 10^{-6} \times 436 \times 10^{-9}}{6.626 \times 10^{-34} \times 2.998 \times 10^{8} \times 6.022 \times 10^{23}}=4.20 \times 10^{-10}
\end{aligned}
$$

Calculated photon flux: $4.20 \times 10^{-10}$ einstein $\cdot \mathrm{s}^{-1}$

(Quantum yield)

A cuvette was charged with a solution of 1,1-ditolyllethylene $2(20.8 \mathrm{mg}, 0.100 \mathrm{mmol}), 3(49.1 \mathrm{mg}, 0.150$ $\mathrm{mmol})$, 1a $(3.4 \mathrm{mg}, 5.00 \mu \mathrm{mol}), 1,2$-dichloroethane $(1.8 \mathrm{~mL})$ and $\mathrm{MeOH}(0.2 \mathrm{~mL})$. The solution was degassed by three freeze-pump-thaw cycles, and it was irradiated by F-7000 ( $\lambda=436 \mathrm{~nm}$, emission slit width $=10.0 \mathrm{~nm}$ ) for $12 \mathrm{~h}$. After evaporation, the yield of 4 was determined by ${ }^{1} \mathrm{H}$ NMR with dimethyl sulfone $(4.71 \mathrm{mg}, 0.0500 \mathrm{mmol})$ as an internal standard.

The quantum yield $(\Phi)$ was calculated by following formula. (A: Absorbance of catalysts)

$$
\phi=\frac{\text { mol product }}{4.20 \times 10^{-10} \cdot 43200 \mathrm{~s} \cdot f} \quad\left(f=1-10^{-A}\right)
$$

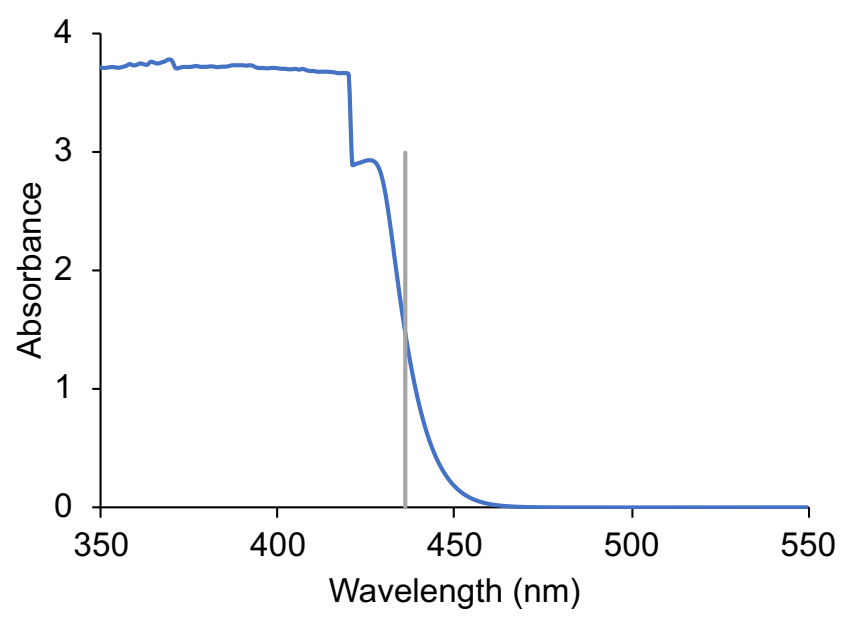

Figure S5. UV-vis spectra of $1 \mathrm{a}(\mathrm{A}=1.4731)$.

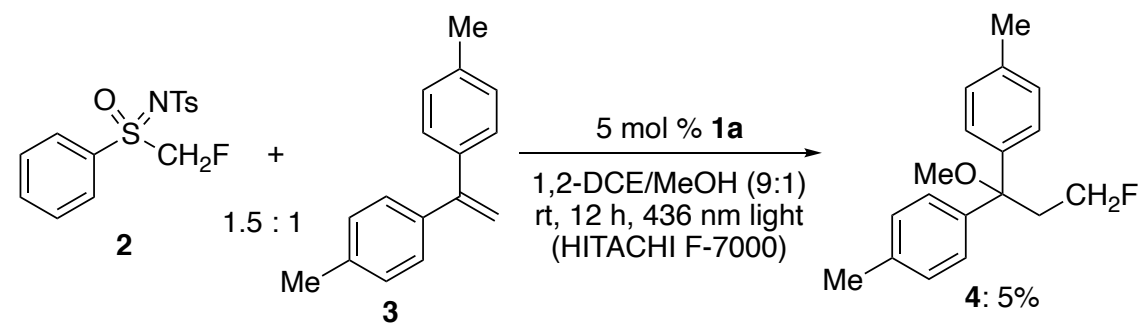

Yield of 4: $5 \%$ (mol product $\left.=5 \times 10^{-6} \mathrm{~mol}\right)$, Calculated quantum yield: $\Phi=\mathbf{2 9} \%$ 


\section{DFT and TD-DFT Calculations of $1 \mathrm{a}, 1 \mathrm{~b}$ and $1 \mathrm{c}$}

Theoretical calculations were performed by using Gaussian 09(D01) and 16 (A03) program package. Ground state geometries of $\mathbf{1 a}, \mathbf{1 b}$ and $1 \mathbf{c}$ were obtained with the CAM-B3LYP/6-31G(d)/CPCMdichloroethane level of theory. Single point TD-DFT calculations were performed with the B3LYP/6$31 \mathrm{G}(\mathrm{d}) / \mathrm{CPCM}$-dichloroethane level of theory. The first three excited states of $\mathbf{1 a}, \mathbf{1 b}$ and $\mathbf{1 c}$ are shown below.

(a) $1 \mathrm{a}$

Excited State 1: $\quad$ Singlet-A $\quad 2.9393 \mathrm{eV} \quad 421.82 \mathrm{~nm} \quad \mathrm{f}=0.2839 \quad<\mathrm{S}^{* *} 2>=0.000$

$$
186>187 \quad 0.70272
$$

Excited State 2: $\quad$ Singlet-A $\quad 3.3174 \mathrm{eV} \quad 373.74 \mathrm{~nm} \quad \mathrm{f}=0.0073 \quad<\mathrm{S}^{* *} 2>=0.000$

$$
185>187 \quad 0.70400
$$

Excited State 3: $\quad$ Singlet-A $\quad 3.6440 \mathrm{eV} \quad 340.25 \mathrm{~nm} \quad \mathrm{f}=0.0731 \quad<S^{* *} 2>=0.000$ $186 \rightarrow 188 \quad 0.69015$

Cartesian coordinates of 1a (6507.6159276032 Hartrees, neutral, singlet).

The Number of Imaginary Frequencies $=0$, Total Energy, $E($ TD-HF/TD-KS $)=-2049.70142105$

$\begin{array}{llcc}\mathrm{N} & 2.84628 & 0.07369 & 0.14237 \\ \mathrm{~N} & -2.82552 & 0.08304 & 0.15132 \\ \mathrm{C} & 2.90887 & -2.22834 & -0.69006 \\ \mathrm{C} & 3.56958 & -3.44041 & -0.82904 \\ \mathrm{C} & 4.86884 & -3.63991 & -0.35173 \\ \mathrm{C} & 5.47281 & -2.55459 & 0.28648 \\ \mathrm{C} & 4.81824 & -1.33971 & 0.45004 \\ \mathrm{C} & 3.52493 & -1.15362 & -0.04298 \\ \mathrm{H} & 1.90634 & -2.11396 & -1.08736 \\ \mathrm{H} & 3.05046 & -4.24787 & -1.3367 \\ \mathrm{H} & 6.47637 & -2.64591 & 0.68525 \\ \mathrm{H} & 5.31787 & -0.527 & 0.96621 \\ \mathrm{C} & 3.51973 & 1.31418 & 0.00576 \\ \mathrm{C} & 3.1299 & 2.40533 & 0.78875 \\ \mathrm{C} & 3.76242 & 3.63046 & 0.65493 \\ \mathrm{C} & 4.81741 & 3.82746 & -0.24514 \\ \mathrm{C} & 5.19862 & 2.72758 & -1.01341 \\ \mathrm{C} & 4.56071 & 1.49547 & -0.90289 \\ \mathrm{H} & 2.32462 & 2.28611 & 1.50627 \\ \mathrm{H} & 3.4273 & 4.45083 & 1.28231 \\ \mathrm{H} & 4.87932 & 0.66962 & -1.52943\end{array}$




\begin{tabular}{|c|c|c|c|}
\hline C & -4.79762 & -1.33223 & 0.45533 \\
\hline C & -5.45155 & -2.5396 & 0.27743 \\
\hline C & -4.85651 & -3.61927 & -0.38931 \\
\hline C & -3.5661 & -3.41742 & -0.87502 \\
\hline C & -2.90116 & -2.20371 & -0.7195 \\
\hline C & -3.50572 & -1.1413 & -0.05089 \\
\hline $\mathrm{H}$ & -5.2881 & -0.52652 & 0.99097 \\
\hline $\mathrm{H}$ & -6.45272 & -2.64284 & 0.68504 \\
\hline $\mathrm{H}$ & -3.05021 & -4.21182 & -1.40165 \\
\hline $\mathrm{H}$ & -1.90208 & -2.08585 & -1.12449 \\
\hline C & -3.09962 & 2.41615 & 0.79589 \\
\hline C & -3.73073 & 3.64217 & 0.66499 \\
\hline C & -4.79075 & 3.84062 & -0.22904 \\
\hline C & -5.17837 & 2.74099 & -0.9942 \\
\hline C & -4.54202 & 1.50767 & -0.88667 \\
\hline C & -3.49613 & 1.32493 & 0.01602 \\
\hline $\mathrm{H}$ & -2.29045 & 2.29598 & 1.50887 \\
\hline $\mathrm{H}$ & -3.39048 & 4.46232 & 1.28989 \\
\hline $\mathrm{H}$ & -5.98706 & 2.83184 & -1.70987 \\
\hline $\mathrm{H}$ & -4.86559 & 0.68243 & -1.51144 \\
\hline C & -5.62039 & -4.93743 & -0.55277 \\
\hline C & -6.91927 & -4.68374 & -1.3388 \\
\hline $\mathrm{H}$ & -7.56869 & -3.96615 & -0.82875 \\
\hline $\mathrm{H}$ & -6.70099 & -4.2898 & -2.33681 \\
\hline $\mathrm{H}$ & -7.48089 & -5.61695 & -1.45679 \\
\hline C & -5.97062 & -5.50469 & 0.83473 \\
\hline $\mathrm{H}$ & -6.52499 & -6.44396 & 0.73222 \\
\hline $\mathrm{H}$ & -5.064 & -5.70664 & 1.41448 \\
\hline $\mathrm{H}$ & -6.59147 & -4.8129 & 1.41153 \\
\hline C & -4.80155 & -5.99092 & -1.30772 \\
\hline $\mathrm{H}$ & -4.54568 & -5.65874 & -2.3189 \\
\hline $\mathrm{H}$ & -3.87293 & -6.23684 & -0.78283 \\
\hline $\mathrm{H}$ & -5.3846 & -6.91269 & -1.39914 \\
\hline C & -5.46361 & 5.21365 & -0.33047 \\
\hline C & -4.42072 & 6.2673 & -0.7448 \\
\hline $\mathrm{H}$ & -4.88766 & 7.25569 & -0.81667 \\
\hline $\mathrm{H}$ & -3.60321 & 6.33698 & -0.02122 \\
\hline $\mathrm{H}$ & -3.98681 & 6.02486 & -1.7204 \\
\hline
\end{tabular}




\begin{tabular}{|c|c|c|c|}
\hline C & -6.5958 & 5.22948 & -1.36402 \\
\hline $\mathrm{H}$ & -7.38838 & 4.51821 & -1.11057 \\
\hline $\mathrm{H}$ & -7.04508 & 6.22685 & -1.40019 \\
\hline $\mathrm{H}$ & -6.23214 & 4.99322 & -2.36912 \\
\hline C & -6.05586 & 5.59781 & 1.03748 \\
\hline $\mathrm{H}$ & -6.81334 & 4.87275 & 1.35271 \\
\hline $\mathrm{H}$ & -5.28757 & 5.64075 & 1.81505 \\
\hline $\mathrm{H}$ & -6.53071 & 6.58351 & 0.98251 \\
\hline C & 5.49205 & 5.19938 & -0.34982 \\
\hline C & 6.09168 & 5.58255 & 1.01519 \\
\hline $\mathrm{H}$ & 6.56811 & 6.56733 & 0.95775 \\
\hline $\mathrm{H}$ & 5.3273 & 5.62706 & 1.79651 \\
\hline $\mathrm{H}$ & 6.84933 & 4.8561 & 1.32681 \\
\hline C & 4.44876 & 6.25468 & -0.75894 \\
\hline $\mathrm{H}$ & 4.91689 & 7.24234 & -0.83302 \\
\hline $\mathrm{H}$ & 4.00967 & 6.013 & -1.73239 \\
\hline $\mathrm{H}$ & 3.63495 & 6.32552 & -0.0313 \\
\hline C & 6.61912 & 5.21342 & -1.38897 \\
\hline $\mathrm{H}$ & 7.41166 & 4.50065 & -1.13963 \\
\hline $\mathrm{H}$ & 6.25006 & 4.97807 & -2.39232 \\
\hline $\mathrm{H}$ & 7.06999 & 6.21 & -1.42715 \\
\hline C & 5.55844 & -4.99612 & -0.53501 \\
\hline C & 4.73988 & -6.08813 & 0.17703 \\
\hline $\mathrm{H}$ & 3.72267 & -6.15545 & -0.21974 \\
\hline $\mathrm{H}$ & 5.21533 & -7.06637 & 0.04581 \\
\hline $\mathrm{H}$ & 4.66961 & -5.88569 & 1.25078 \\
\hline C & 5.64923 & -5.3292 & -2.03516 \\
\hline $\mathrm{H}$ & 4.66073 & -5.37636 & -2.50128 \\
\hline $\mathrm{H}$ & 6.23699 & -4.57463 & -2.5681 \\
\hline $\mathrm{H}$ & 6.13281 & -6.30146 & -2.18 \\
\hline C & 6.97787 & -5.00726 & 0.04417 \\
\hline $\mathrm{H}$ & 7.43045 & -5.99139 & -0.11271 \\
\hline $\mathrm{H}$ & 7.62063 & -4.26552 & -0.44069 \\
\hline $\mathrm{H}$ & 6.97943 & -4.81 & 1.12095 \\
\hline $\mathrm{H}$ & 6.00327 & 2.81751 & -1.73372 \\
\hline C & -0.69467 & -1.60797 & 3.43333 \\
\hline C & -1.38452 & -1.06966 & 2.37972 \\
\hline C & -0.70134 & -0.51135 & 1.26913 \\
\hline
\end{tabular}




$\begin{array}{lccl}\mathrm{C} & 0.72303 & -0.51328 & 1.26778 \\ \mathrm{C} & 1.40653 & -1.07254 & 2.37778 \\ \mathrm{C} & 0.71692 & -1.60917 & 3.43243 \\ \mathrm{H} & -1.23352 & -2.02587 & 4.27781 \\ \mathrm{H} & -2.46768 & -1.0538 & 2.38683 \\ \mathrm{C} & -1.40049 & 0.08717 & 0.17411 \\ \mathrm{C} & 1.42151 & 0.08196 & 0.17036 \\ \mathrm{H} & 2.48971 & -1.05931 & 2.38377 \\ \mathrm{H} & 1.2561 & -2.0278 & 4.27633 \\ \mathrm{C} & 0.71392 & 0.68 & -0.84222 \\ \mathrm{C} & -0.6936 & 0.68302 & -0.84016 \\ \mathrm{H} & 1.2504 & 1.13829 & -1.66633 \\ \mathrm{H} & -1.23054 & 1.14399 & -1.6625\end{array}$

(b) $\mathbf{1 b}$

Excited State 1: Singlet-A $\quad 3.0927$ eV $\quad 400.89 \mathrm{~nm} \quad \mathrm{f}=0.2588 \quad<\mathrm{S}^{* *} 2>=0.000$ $186->187 \quad 0.70112$

Excited State 2: $\quad$ Singlet-A $\quad 3.1311 \mathrm{eV} \quad 395.98 \mathrm{~nm} \quad \mathrm{f}=0.0014 \quad<\mathrm{S}^{\star \star} 2>=0.000$ $185->187 \quad 0.70441$

Excited State 3: Singlet-A $\quad 3.7915 \mathrm{eV} \quad 327.01 \mathrm{~nm} \quad \mathrm{f}=0.0753 \quad<\mathrm{S}^{* *} 2>=0.000$ $186->188 \quad 0.67893$

Cartesian coordinates of $\mathbf{1 b}$ (6452.9097330243 Hartrees, neutral, singlet).

The Number of Imaginary Frequencies = 0, Total Energy, E (TD-HF/TD-KS $)=-2049.69541143$

$\begin{array}{lrll}\text { C } & 1.5976 & -0.7786 & 1.80125 \\ \text { C } & 1.78421 & -0.13281 & 0.60396 \\ \text { C } & 0.66276 & 0.17481 & -0.22956 \\ \text { C } & -0.64112 & -0.1813 & 0.22144 \\ \text { C } & -0.79203 & -0.828 & 1.47425 \\ \text { C } & 0.30401 & -1.1292 & 2.23706 \\ \text { H } & 1.80817 & 1.07396 & -1.82836 \\ \text { H } & 2.46046 & -1.00798 & 2.41781 \\ \text { C } & 0.81356 & 0.82076 & -1.48288 \\ \text { C } & -1.76276 & 0.12753 & -0.61133 \\ \text { H } & -1.78671 & -1.08145 & 1.81927 \\ \text { H } & 0.18186 & -1.62412 & 3.19513 \\ \text { C } & -1.57636 & 0.77279 & -1.80886 \\ \text { C } & -0.28257 & 1.12192 & -2.24553\end{array}$




\begin{tabular}{|c|c|c|c|}
\hline $\mathrm{H}$ & -2.43935 & 1.00331 & -2.42482 \\
\hline $\mathrm{H}$ & -0.16046 & 1.61618 & -3.20395 \\
\hline$N$ & -3.07842 & -0.22667 & -0.18847 \\
\hline$N$ & 3.10022 & 0.22278 & 0.18359 \\
\hline C & -4.05262 & 0.80084 & -0.10889 \\
\hline C & -5.32998 & 0.65156 & -0.65629 \\
\hline C & -6.25644 & 1.67957 & -0.56737 \\
\hline & -5.95703 & 2.90135 & 0.04645 \\
\hline C & -4.67344 & 3.03799 & 0.57732 \\
\hline C & -3.7399 & 2.00981 & 0.51144 \\
\hline $\mathrm{H}$ & -5.598 & -0.27371 & -1.15432 \\
\hline $\mathrm{H}$ & -7.23717 & 1.52196 & -1.00612 \\
\hline $\mathrm{H}$ & -4.38079 & 3.95861 & 1.0684 \\
\hline $\mathrm{H}$ & -2.75504 & 2.14727 & 0.94599 \\
\hline C & -3.45149 & -1.59072 & -0.16349 \\
\hline C & -2.81649 & -2.53123 & -0.97214 \\
\hline C & -3.16565 & -3.8777 & -0.90634 \\
\hline C & -4.16402 & -4.34112 & -0.05165 \\
\hline C & -4.79475 & -3.38214 & 0.7524 \\
\hline C & -4.44771 & -2.04233 & 0.71164 \\
\hline $\mathrm{H}$ & -2.04193 & -2.21323 & -1.66124 \\
\hline $\mathrm{H}$ & -2.63925 & -4.56759 & -1.55556 \\
\hline $\mathrm{H}$ & -5.57352 & -3.68618 & 1.44548 \\
\hline $\mathrm{H}$ & -4.94973 & -1.3355 & 1.36357 \\
\hline C & 4.47359 & 2.03598 & -0.71343 \\
\hline C & 4.82075 & 3.3808 & -0.76088 \\
\hline C & 4.17717 & 4.33884 & 0.02498 \\
\hline C & 3.1664 & 3.87451 & 0.87268 \\
\hline C & 2.81994 & 2.53308 & 0.94564 \\
\hline C & 3.47046 & 1.58692 & 0.14854 \\
\hline $\mathrm{H}$ & 4.98782 & 1.32757 & -1.35396 \\
\hline $\mathrm{H}$ & 5.60814 & 3.67561 & -1.44485 \\
\hline $\mathrm{H}$ & 2.63203 & 4.57306 & 1.50974 \\
\hline $\mathrm{H}$ & 2.03851 & 2.21641 & 1.62748 \\
\hline C & 5.34946 & -0.6608 & 0.653 \\
\hline C & 6.27425 & -1.69001 & 0.56276 \\
\hline 0 & 5.97472 & -2.90852 & -0.05783 \\
\hline & 4.69301 & -3.04037 & -0.59409 \\
\hline
\end{tabular}




\begin{tabular}{|c|c|c|c|}
\hline C & 3.7611 & -2.0106 & -0.52678 \\
\hline C & 4.07376 & -0.80537 & 0.1004 \\
\hline $\mathrm{H}$ & 5.61732 & 0.26203 & 1.15563 \\
\hline $\mathrm{H}$ & 7.25371 & -1.53634 & 1.0057 \\
\hline $\mathrm{H}$ & 4.40044 & -3.95813 & -1.09051 \\
\hline $\mathrm{H}$ & 2.77748 & -2.14419 & -0.96536 \\
\hline C & -4.57822 & -5.81361 & 0.03749 \\
\hline C & -7.01303 & 4.01021 & 0.10755 \\
\hline C & 4.52931 & 5.82987 & -0.0119 \\
\hline C & 7.02903 & -4.01888 & -0.12024 \\
\hline C & 3.29075 & 6.64066 & -0.4335 \\
\hline $\mathrm{H}$ & 3.52685 & 7.71018 & -0.458 \\
\hline $\mathrm{H}$ & 2.95095 & 6.34466 & -1.4314 \\
\hline $\mathrm{H}$ & 2.45753 & 6.49895 & 0.261 \\
\hline C & 5.65946 & 6.13316 & -1.00254 \\
\hline $\mathrm{H}$ & 5.38748 & 5.85723 & -2.02638 \\
\hline $\mathrm{H}$ & 5.87604 & 7.20603 & -0.9949 \\
\hline $\mathrm{H}$ & 6.58266 & 5.60676 & -0.73995 \\
\hline C & 4.98094 & 6.28628 & 1.38704 \\
\hline $\mathrm{H}$ & 5.86981 & 5.73435 & 1.70971 \\
\hline $\mathrm{H}$ & 5.22827 & 7.35347 & 1.37727 \\
\hline $\mathrm{H}$ & 4.19893 & 6.13209 & 2.13627 \\
\hline C & 6.51286 & -5.26862 & -0.84292 \\
\hline $\mathrm{H}$ & 5.64396 & -5.70177 & -0.33721 \\
\hline $\mathrm{H}$ & 6.23373 & -5.05305 & -1.87923 \\
\hline $\mathrm{H}$ & 7.2981 & -6.03078 & -0.86291 \\
\hline C & 8.27049 & -3.50793 & -0.87336 \\
\hline $\mathrm{H}$ & 9.03533 & -4.29087 & -0.91953 \\
\hline $\mathrm{H}$ & 8.01512 & -3.22163 & -1.89887 \\
\hline $\mathrm{H}$ & 8.71448 & -2.63726 & -0.38188 \\
\hline C & 7.43394 & -4.423 & 1.30872 \\
\hline $\mathrm{H}$ & 6.57192 & -4.80458 & 1.86563 \\
\hline $\mathrm{H}$ & 8.1953 & -5.21012 & 1.27916 \\
\hline $\mathrm{H}$ & 7.84859 & -3.57886 & 1.86738 \\
\hline C & -6.06987 & -5.95032 & -0.31708 \\
\hline $\mathrm{H}$ & -6.26237 & -5.6024 & -1.33726 \\
\hline $\mathrm{H}$ & -6.70302 & -5.37073 & 0.36108 \\
\hline & -6.38177 & -6.99837 & -0.25063 \\
\hline
\end{tabular}




$\begin{array}{llll}\mathrm{C} & -3.77501 & -6.70015 & -0.92139 \\ \mathrm{H} & -3.9167 & -6.40284 & -1.96536 \\ \mathrm{H} & -2.70332 & -6.67196 & -0.69996 \\ \mathrm{H} & -4.10604 & -7.73888 & -0.8245 \\ \mathrm{C} & -4.34969 & -6.32604 & 1.47085 \\ \mathrm{H} & -3.29273 & -6.25376 & 1.74739 \\ \mathrm{H} & -4.92871 & -5.75565 & 2.20303 \\ \mathrm{H} & -4.65145 & -7.37611 & 1.5518 \\ \mathrm{C} & -7.42252 & 4.40816 & -1.32185 \\ \mathrm{H} & -7.83686 & 3.56105 & -1.87623 \\ \mathrm{H} & -6.5627 & 4.78936 & -1.88241 \\ \mathrm{H} & -8.18539 & 5.19386 & -1.2933 \\ \mathrm{C} & -6.49693 & 5.26356 & 0.82399 \\ \mathrm{H} & -7.28349 & 6.02436 & 0.84358 \\ \mathrm{H} & -5.63039 & 5.69639 & 0.31399 \\ \mathrm{H} & -6.2142 & 5.05227 & 1.86021 \\ \mathrm{C} & -8.25161 & 3.50023 & 0.86605 \\ \mathrm{H} & -9.0174 & 4.28227 & 0.91168 \\ \mathrm{H} & -8.69586 & 2.62723 & 0.37897 \\ \mathrm{H} & -7.99289 & 3.2179 & 1.89183 \\ & & & \\ & & & \\ & & & \end{array}$

(c) $1 \mathrm{c}$

Excited State 1: $\quad$ Singlet-A $\quad 3.2033 \mathrm{eV} \quad 387.05 \mathrm{~nm} \quad \mathrm{f}=0.1221 \quad<\mathrm{S}^{* \star} 2>=0.000$

$$
110 \rightarrow 111 \quad 0.70216
$$

Excited State 2: $\quad$ Singlet-A $\quad 3.8754 \mathrm{eV} \quad 319.93 \mathrm{~nm} \quad \mathrm{f}=0.0504 \quad<\mathrm{S}^{* *} 2>=0.000$

$$
110->112 \quad 0.69196
$$

Excited State $\quad 3: \quad$ Singlet-A $\quad 4.1970 \mathrm{eV} \quad 295.41 \mathrm{~nm} \quad \mathrm{f}=0.5169 \quad<S^{* *} 2>=0.000$

$$
\begin{array}{ll}
110->113 & 0.68810 \\
110->114 & 0.12085
\end{array}
$$

Cartesian coordinates of 1c (2907.3859691806 Hartrees, neutral, singlet).

The Number of Imaginary Frequencies $=0$, Total Energy, E $($ TD-HF/TD-KS $)=-1217.73486821$

$\begin{array}{lllr}\text { C } & -2.28621 & 4.5903 & -1.71637 \\ \text { C } & -1.73513 & 4.98005 & -0.52545 \\ \text { C } & -0.97975 & 4.07202 & 0.26118 \\ \text { C } & -0.81208 & 2.73522 & -0.19841 \\ \text { C } & -1.38251 & 2.36536 & -1.44372 \\ \text { C } & -2.09994 & 3.26899 & -2.18262\end{array}$




\begin{tabular}{|c|c|c|c|}
\hline $\mathrm{H}$ & -0.50521 & 5.50138 & 1.81295 \\
\hline $\mathrm{H}$ & -2.86087 & 5.29468 & -2.30948 \\
\hline $\mathrm{H}$ & -1.8635 & 5.99639 & -0.1639 \\
\hline$C$ & -0.37497 & 4.47622 & 1.47892 \\
\hline & -0.05763 & 1.82481 & 0.60683 \\
\hline $\mathrm{H}$ & -1.23849 & 1.35502 & -1.80756 \\
\hline $\mathrm{H}$ & -2.52654 & 2.97129 & -3.13535 \\
\hline & 0.51611 & 2.25631 & 1.7782 \\
\hline C & 0.36621 & 3.59044 & 2.21282 \\
\hline $\mathrm{H}$ & 1.09009 & 1.55406 & 2.37384 \\
\hline $\mathrm{H}$ & 0.83137 & 3.90142 & 3.14273 \\
\hline$N$ & 0.11205 & 0.47383 & 0.18295 \\
\hline C & 1.7906 & -1.27576 & 0.60624 \\
\hline C & 3.09216 & -1.73989 & 0.49 \\
\hline C & 4.09619 & -0.99298 & -0.13711 \\
\hline C & 3.72341 & 0.24927 & -0.65267 \\
\hline C & 2.41839 & 0.71985 & -0.55964 \\
\hline C & 1.43156 & -0.03402 & 0.07435 \\
\hline $\mathrm{H}$ & 1.04655 & -1.88008 & 1.11331 \\
\hline $\mathrm{H}$ & 3.32804 & -2.70984 & 0.91739 \\
\hline $\mathrm{H}$ & 4.45383 & 0.87402 & -1.15316 \\
\hline $\mathrm{H}$ & 2.16195 & 1.68562 & -0.98287 \\
\hline C & -2.10736 & -0.16643 & 0.99631 \\
\hline C & -3.21501 & -1.00004 & 0.94062 \\
\hline C & -3.27991 & -2.09687 & 0.07535 \\
\hline C & -2.17054 & -2.31077 & -0.74527 \\
\hline C & -1.06069 & -1.475 & -0.7145 \\
\hline C & -1.0073 & -0.39058 & 0.16394 \\
\hline $\mathrm{H}$ & -2.09466 & 0.66502 & 1.69229 \\
\hline $\mathrm{H}$ & -4.04684 & -0.78611 & 1.60519 \\
\hline $\mathrm{H}$ & -2.16034 & -3.13934 & -1.44375 \\
\hline $\mathrm{H}$ & -0.22659 & -1.66727 & -1.38081 \\
\hline C & 5.5224 & -1.54614 & -0.23023 \\
\hline C & -4.52264 & -2.99338 & 0.05737 \\
\hline C & 6.47198 & -0.58274 & -0.95176 \\
\hline $\mathrm{H}$ & 6.55107 & 0.37702 & -0.43123 \\
\hline & 7.47445 & -1.01988 & -0.99483 \\
\hline & 6.15062 & -0.38904 & -1.98015 \\
\hline
\end{tabular}




$\begin{array}{llll}\mathrm{C} & 5.50838 & -2.87619 & -1.00497 \\ \mathrm{H} & 5.13194 & -2.73142 & -2.02291 \\ \mathrm{H} & 6.52165 & -3.28695 & -1.07265 \\ \mathrm{H} & 4.87727 & -3.62411 & -0.51626 \\ \mathrm{C} & 6.07156 & -1.79111 & 1.18675 \\ \mathrm{H} & 5.45727 & -2.50549 & 1.74255 \\ \mathrm{H} & 7.08872 & -2.19468 & 1.1353 \\ \mathrm{H} & 6.10459 & -0.85844 & 1.75937 \\ \mathrm{C} & -4.39882 & -4.13388 & -0.95975 \\ \mathrm{H} & -3.55133 & -4.78914 & -0.73457 \\ \mathrm{H} & -4.27861 & -3.7571 & -1.98051 \\ \mathrm{H} & -5.30592 & -4.74596 & -0.93735 \\ \mathrm{C} & -5.75823 & -2.15257 & -0.31109 \\ \mathrm{H} & -6.65646 & -2.77969 & -0.31921 \\ \mathrm{H} & -5.64563 & -1.70744 & -1.30511 \\ \mathrm{H} & -5.92447 & -1.34133 & 0.40359 \\ \mathrm{C} & -4.72929 & -3.61287 & 1.45135 \\ \mathrm{H} & -4.86622 & -2.8465 & 2.21994 \\ \mathrm{H} & -5.61895 & -4.25219 & 1.45519 \\ \mathrm{H} & -3.86876 & -4.22649 & 1.73735\end{array}$

\section{Comparison of UV-Vis Spectra and Transitions Predicted by TD-DFT}

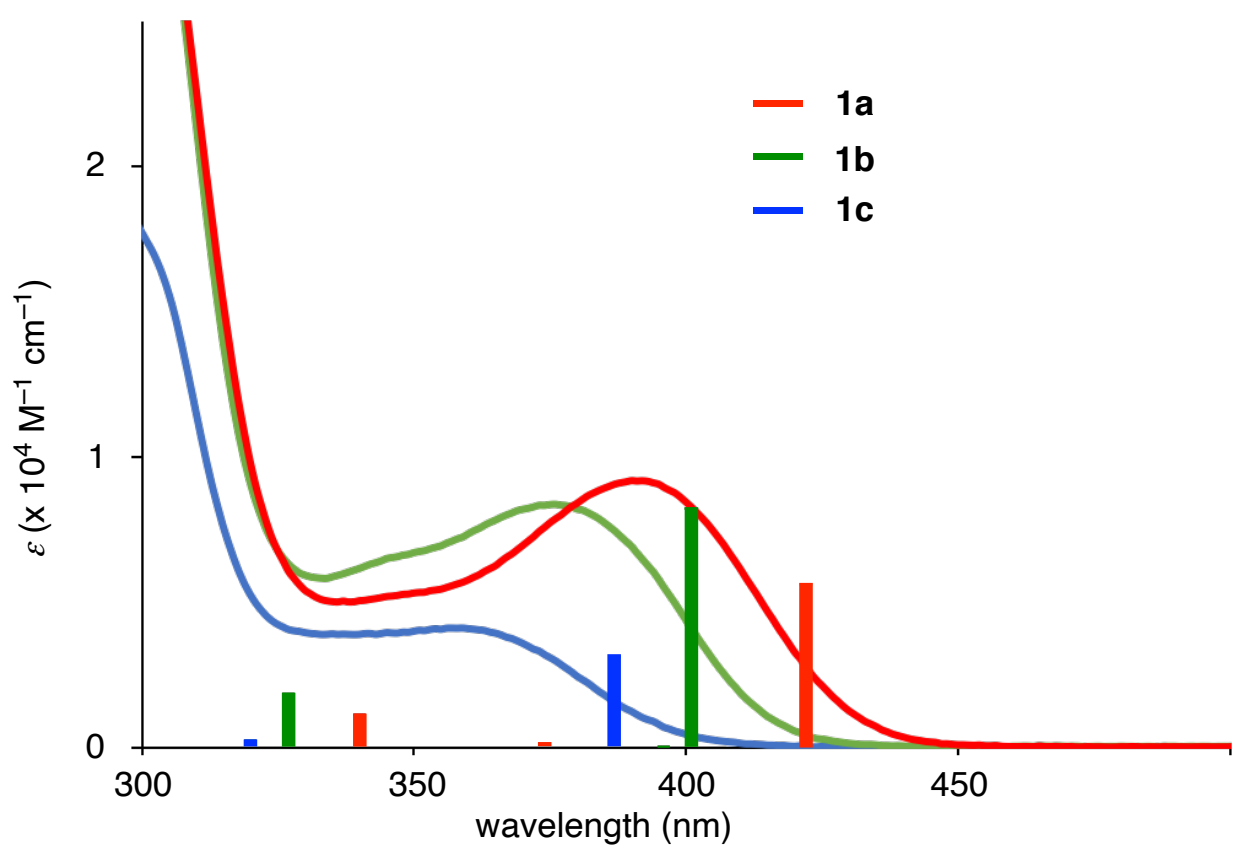

Figure S6. UV-Vis spectra (1a-c) and transitions predicted by TD-DFT calculation (bars) 


\section{NMR Spectra}

${ }^{1} \mathrm{H}$ NMR (400 MHz, $\left.\mathrm{CD}_{2} \mathrm{Cl}_{2}, \mathrm{rt}\right)$

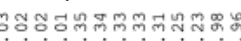

$\bigcup^{\infty}$

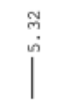

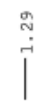

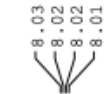

||

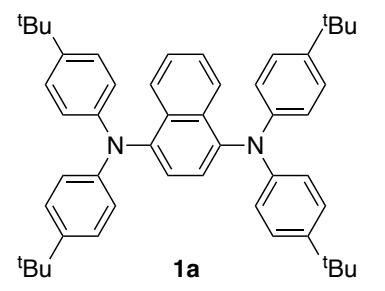

$1 a$

'Bu

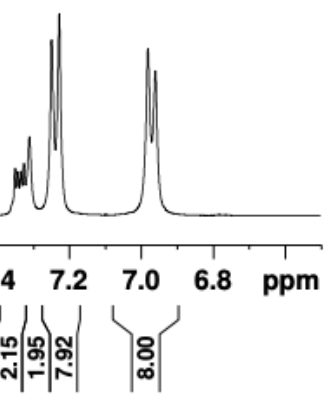

di

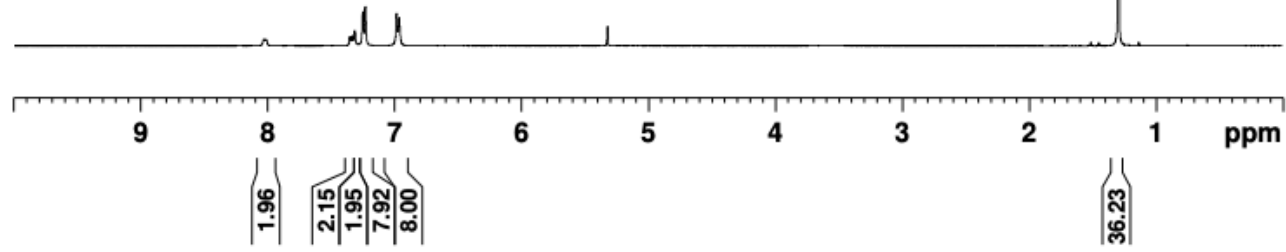

${ }^{13} \mathrm{C}\left\{{ }^{1} \mathrm{H}\right\}$ NMR $\left(126 \mathrm{MHz}, \mathrm{CD}_{2} \mathrm{Cl} 2, \mathrm{rt}\right)$
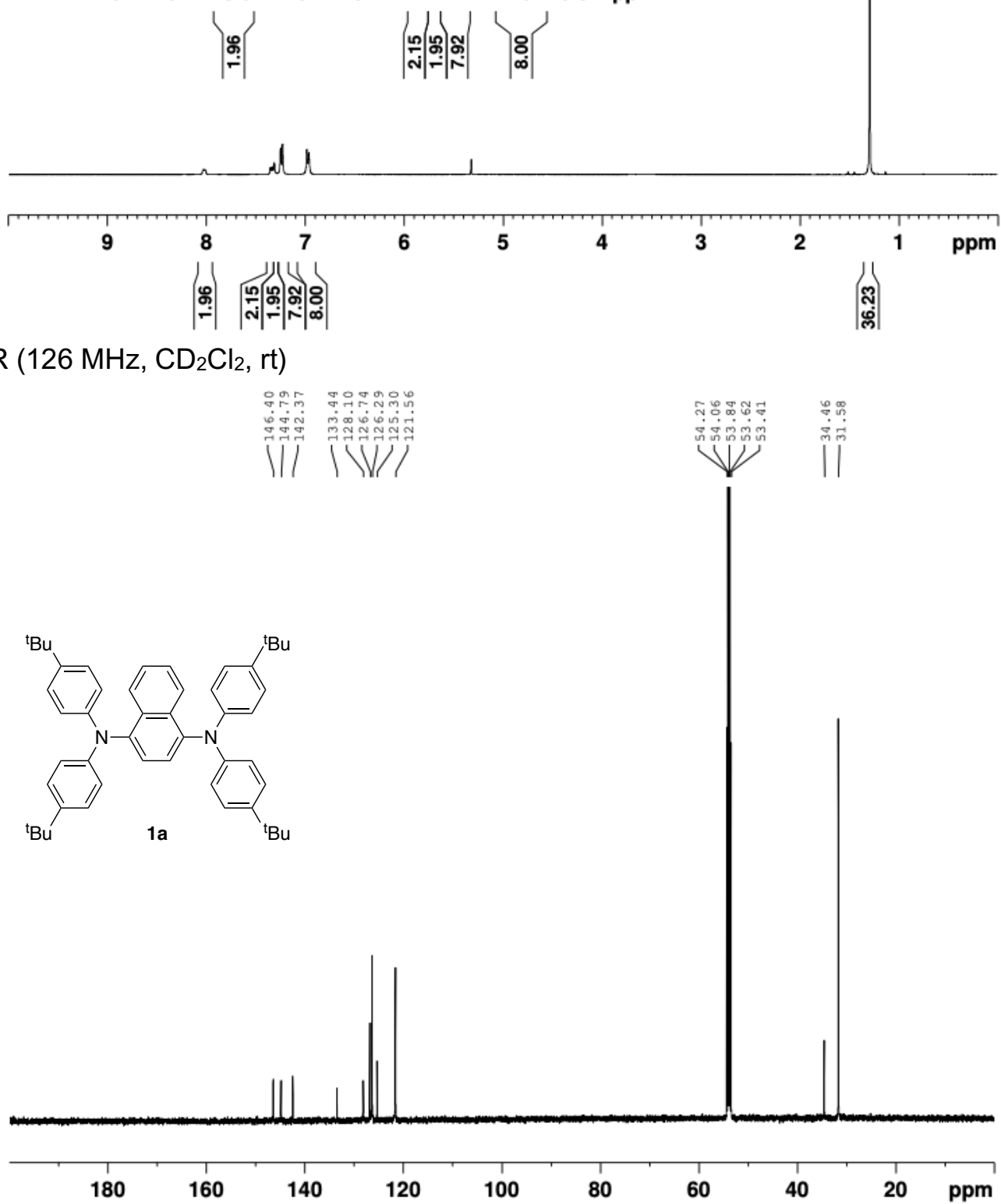
${ }^{1} \mathrm{H}$ NMR $\left(400 \mathrm{MHz}, \mathrm{CDCl}_{3}, \mathrm{rt}\right)$

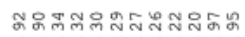

$\underbrace{2}$

'Bu<smiles>Brc1ccc(N(c2ccc(Br)cc2)c2cccc3c(N(c4ccc(Br)cc4)c4ccc(Br)cc4)cccc23)cc1</smiles>

VI

$\underbrace{n}$

${ }^{\mathrm{t} B u}$

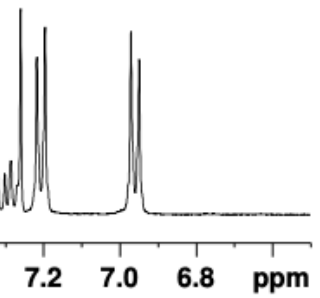

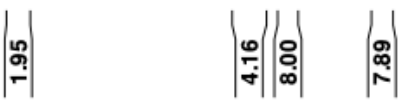

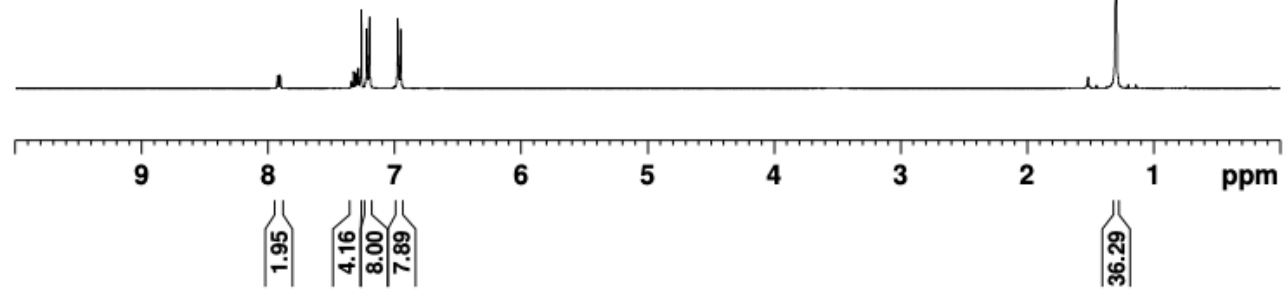

${ }^{13} \mathrm{C}\left\{{ }^{1} \mathrm{H}\right\}$ NMR $\left(126 \mathrm{MHz}, \mathrm{CDCl}_{3}, \mathrm{rt}\right)$

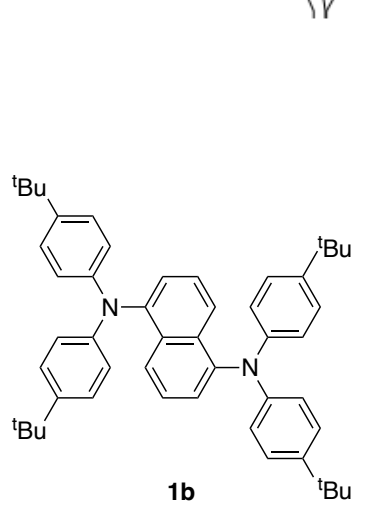

|V

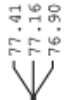

|்ֶ

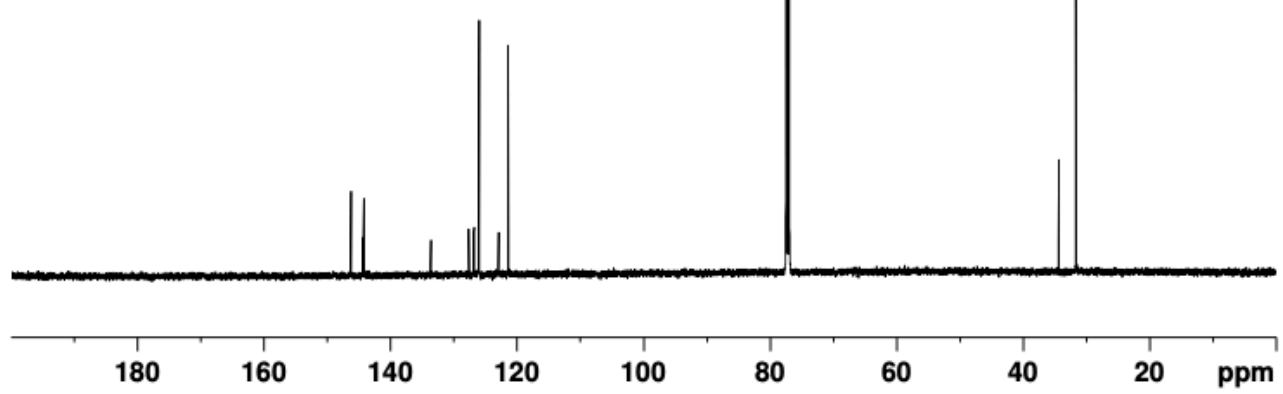


${ }^{1} \mathrm{H}$ NMR $\left(400 \mathrm{MHz}, \mathrm{CDCl}_{3}, \mathrm{rt}\right)$
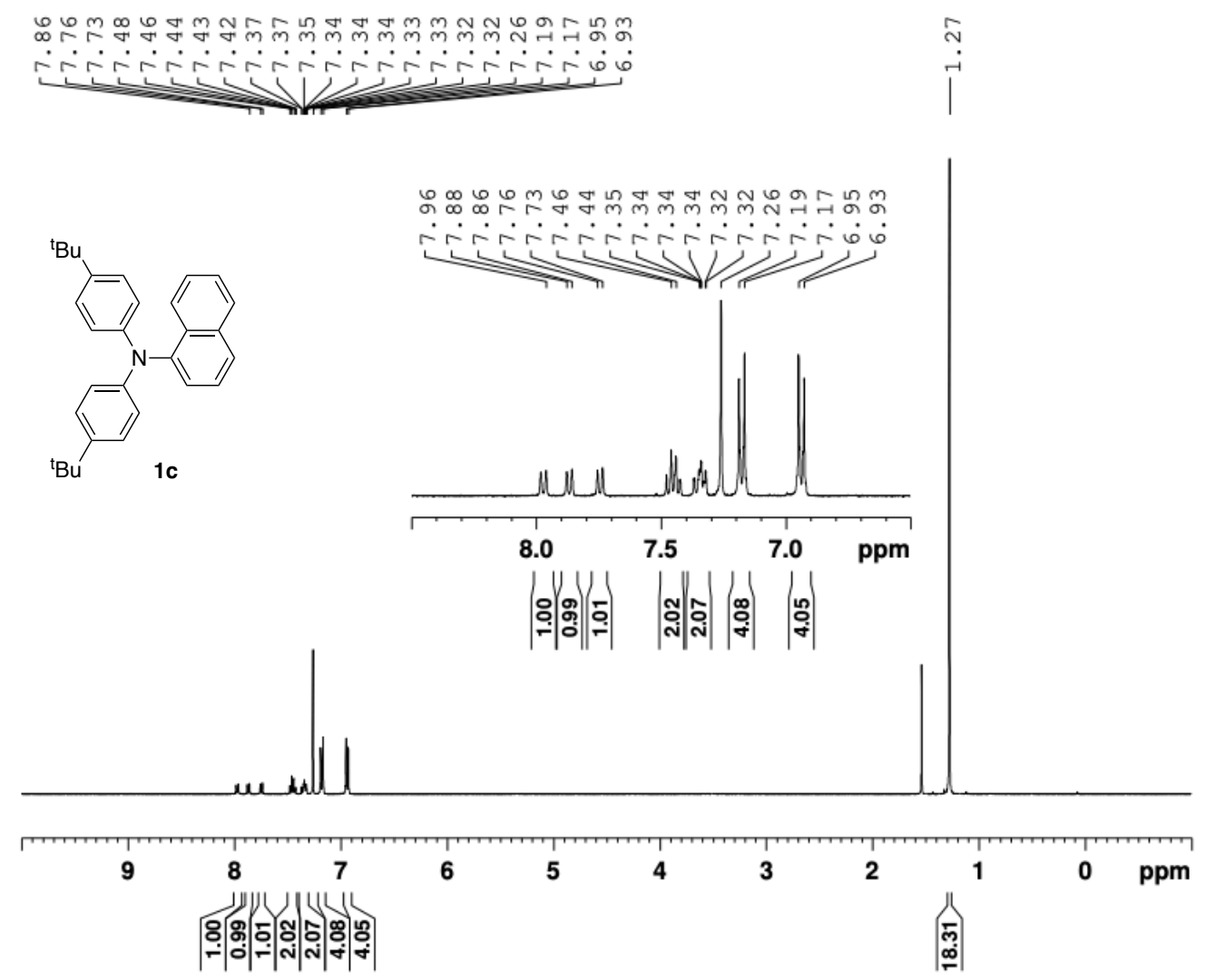

${ }^{1} \mathrm{H}$ NMR $\left(400 \mathrm{MHz}, \mathrm{CDCl}_{3}, \mathrm{rt}\right)$

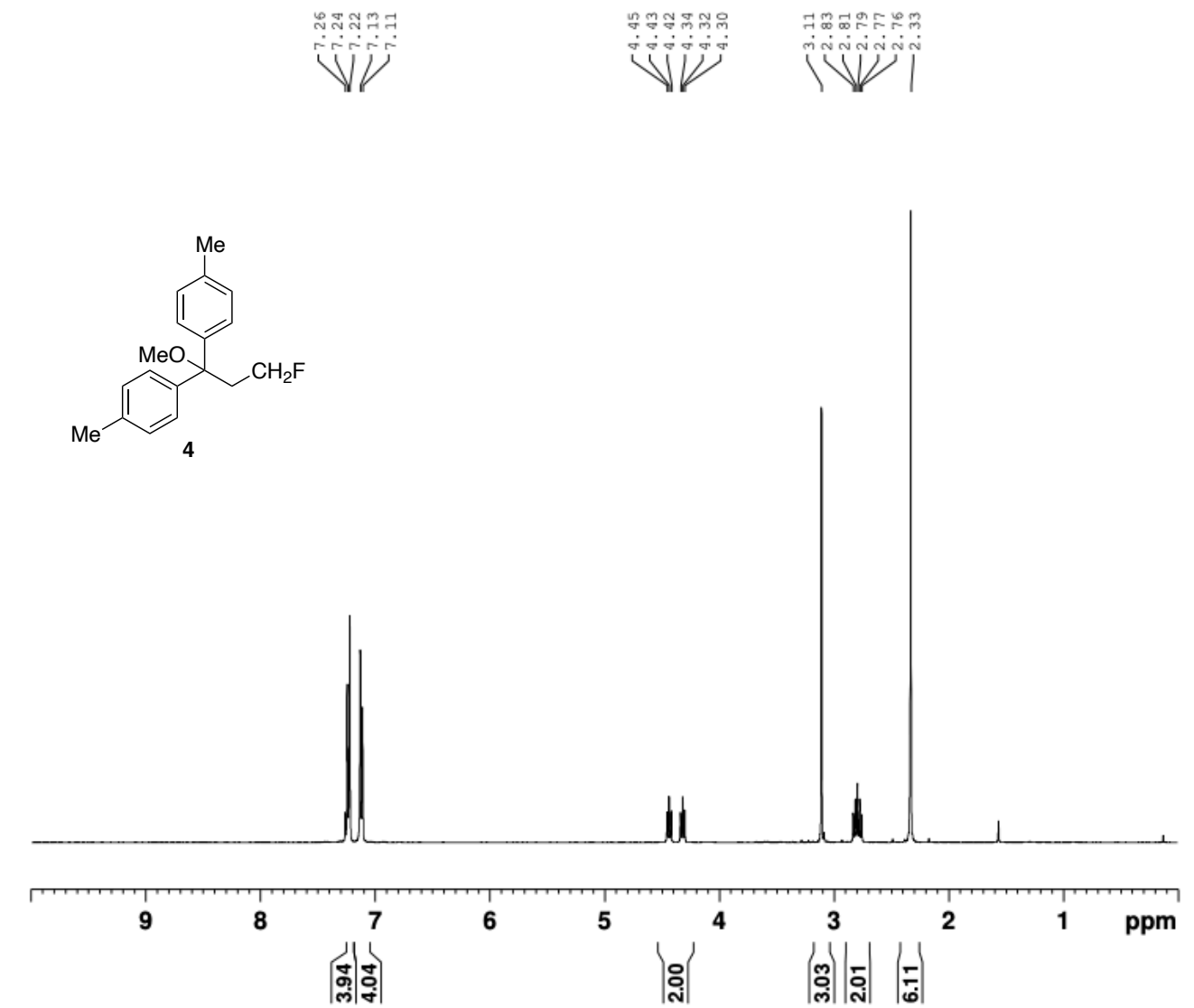


${ }^{13} \mathrm{C}\left\{{ }^{1} \mathrm{H}\right\} \operatorname{NMR}\left(126 \mathrm{MHz}, \mathrm{CDCl}_{3}, \mathrm{rt}\right)$
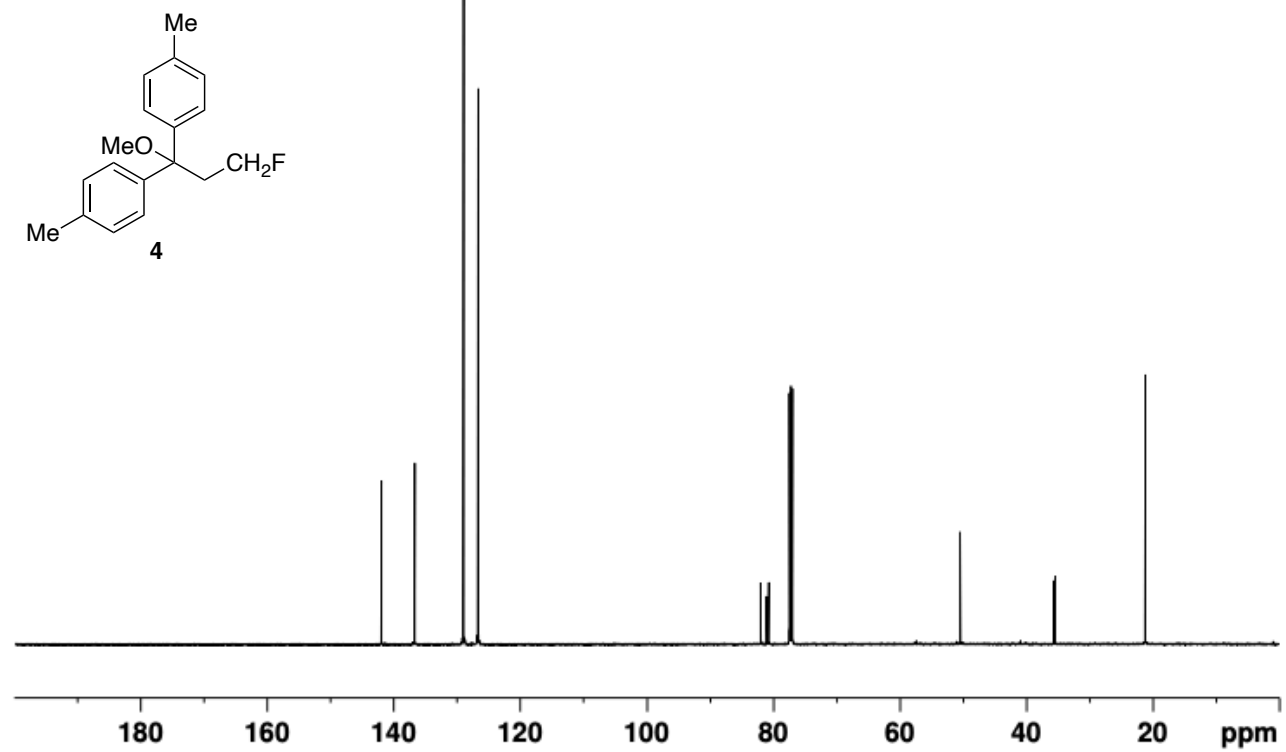

${ }^{19} \mathrm{~F} \mathrm{NMR}\left(376 \mathrm{MHz}, \mathrm{CDCl}_{3}, \mathrm{rt}\right)$

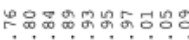

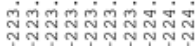

an

용ํำ

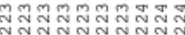

vilivilil
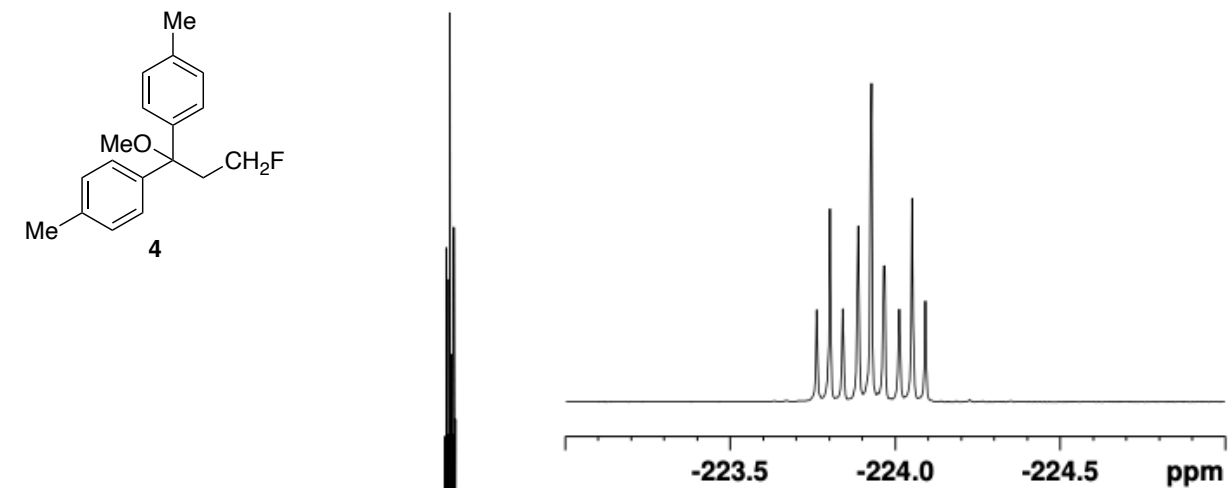

$-215$

$-220$

$-225$

$-230$

$-235$

$-240$

$-245$

ppm 